\title{
Thirty-six combined years of MODIS geolocation trending
}

\author{
Guoqing (Gary) Lin*a,b, Robert E. Wolfe ${ }^{\mathrm{b}}$, Ping Zhang ${ }^{\mathrm{a}, \mathrm{b}}$, \\ James C. Tilton ${ }^{\mathrm{b}}$, John J. Dellomo ${ }^{\mathrm{c}, \mathrm{b}}$, Bin Tan ${ }^{\mathrm{a}, \mathrm{b}}$ \\ ${ }^{a}$ Science Systems and Applications, Inc., 10210 Greenbelt Road, Lanham, MD 20706, USA; \\ ${ }^{b}$ NASA Goddard Space Flight Center, 8800 Greenbelt Rd, Greenbelt, MD 20771, USA; \\ ${ }^{\mathrm{c}}$ Global Science \& Technology, Inc., 7855 Walker Drive, Greenbelt, MD 20770, USA
}

\begin{abstract}
Two Moderate Resolution Imaging Spectroradiometer (MODIS) sensors have been in operations for more than 19 and 17 years (thus 36 combined years) as part of NASA's Earth Observing System (EOS) on the Terra platform that was launched in December 1999 and on the Aqua platform that was launched in May 2002, respectively. Accurate geolocation is a critical element needed for accurate retrieval of global biogeophysical parameters. In this paper, we describe the latest trends in the continuously improved MODIS geolocation accuracy in Collection-5 (C5), C6 and C6.1 re-processing and forward-processing data streams. We improved geolocation accuracy in the re-processed data and corrected for geolocation biases found in forward-processed data, including those caused by operations such as the stop-go-stop status of the Advanced Microwave Scanning Radiometer for EOS (AMSR-E) instrument on the Aqua platform. We discuss scan-toscan underlaps near nadir over the equator regions that was discovered in checking the non-underlapping requirement in the Visible Infrared Imaging Radiometer Suite (VIIRS) based on trending parameters from the actual Suomi National Polar-orbiting Partnership (S-NPP) satellite orbit. The underlaps are closely tied to instrument effective focal length that is measured from on-orbit data using a technique we recently developed. We also discuss potential improvements for the upcoming $\mathrm{C} 7$ re-processing.
\end{abstract}

Keywords: MODIS, Terra, Aqua, geolocation, ground control point, error analysis, scan-to-scan underlaps, focal length

\section{INTRODUCTION}

Two Moderate Resolution Imaging Spectroradiometer (MODIS) sensors have been in operations as part of NASA's Earth Observing System (EOS) since 1999. The first one was launched onboard Terra platform on 18 December 1999 while the second one on Aqua was launched on 4 May 2002. Data products from Terra MODIS are available from 24 February 2000 while the products from Aqua MODIS are available from 4 July 2002. The combined data products are available for more than 36 combined years at the time of this writing in August 2019.

On-orbit MODIS geolocation calibration and validation have gone through many years of improvements. The progresses in the geolocation monitoring and error corrections may be found in status reports at meetings [1,2] and publications (e.g., those in websites $[3,4]$, and specific ones in $[5,6,7,8,9]$ ). (The oldest meeting reports were from 1998 and the oldest publication was from 1987 that was 12 years before the first MODIS was launched.) The details of the MODIS instruments may be found in [10]. The theory for ground geolocation software is described in [11]

At the time of implementing the geolocation product, it was decided that the geolocation products were to be represented by the coarsest resolution one kilometer $(1 \mathrm{KM})$ "ideal" band [11]. There are a total of 36 bands on four focal plane assemblies (FPAs): two (2) quarter kilometer (QKM) bands on near-infrared (NIR) FPA, five (5) half-kilometer (HKM) bands on visible (VIS) FPA and short/mid-wave IR (S/MWIR) FPA, and 29 1KM bands distributed in all 4 FPAs (Fig. 1).

*Gary.Lin@ @asa.gov; glin@ssaihq.com; phone 301-614-5451; fax 301-614-5269; www.ssai.org. 


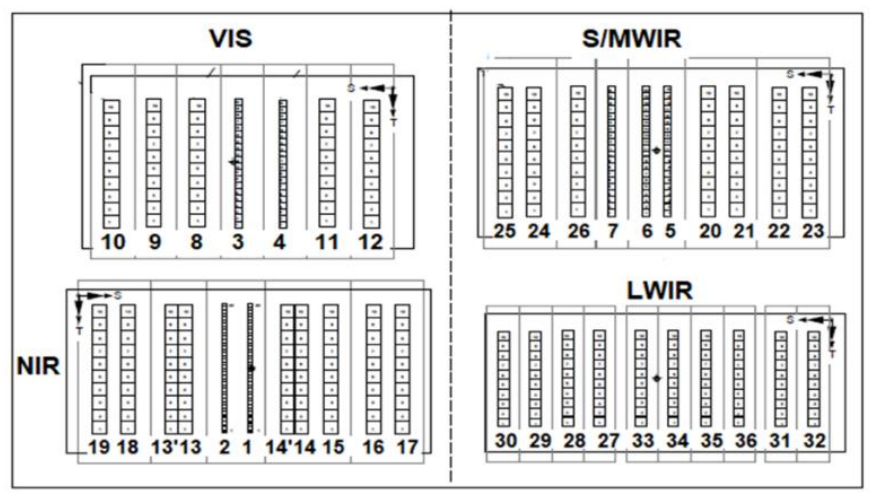

Fig. 1. Schematics of the MODIS bands on 4 FPAs.

On-orbit geolocation errors are measured using a ground Control Point Matching (CPM) program. Each day for each MODIS instrument, the CPM program runs over 1000 globally distributed Landsat ground control point (GCP) image chips to MODIS Band 1 image. Each pre-selected and cloud-free $30 \mathrm{~m}$ resolution Landsat red band chip is used to simulate MODIS QKM band 1 data by using a first order point spread function as a weighting function. Geolocation error is determined by moving Landsat location until the correlation between the simulated data and MODIS data is at its maximum. When a good match is obtained ( 250 matches/day), the measured shift is used in the error analysis. The details of the CPM is described in [5, 9].

The measured geolocation errors are "stratified" mainly in time, scan angle, and sun angle with respect to spacecraft velocity. After a large number of measurements are obtained, they are stratified to form biases. Models were then constructed to correct for the biases [9]. This is possible because we have accurate knowledge of orbit state (position and velocity) and spacecraft orientation (attitude) of the Terra and Aqua satellites, good prelaunch geometric instrument characterization, accurate geometric models of the instrument and the earth terrain, and accurate GCP reference data because of the Landsat program $[12,13]$.

The as-built geometry of the two MODIS sensors are almost identical. They are onboard two different platforms, one on Terra [14] and another on Aqua [15] as shown in Fig. 2. Note that the MODIS sensors are mounted at the nadir deck on both platforms. However, on the Aqua platform, the Advanced Microwave Scanning Radiometer for EOS (AMSR-E) sensor is mounted at the zenith deck directly above the MODIS sensor. We will see later that the activity of the AMSR-E sensor impacts on MODIS pointing and thus the geolocation error patterns. Another difference is the day-time orbits: Terra is in the morning with a nominal local time descending node (LTDN) at 10:30 AM while Aqua is in the afternoon with a nominal local time ascending node (LTAN) at 1:35 PM.
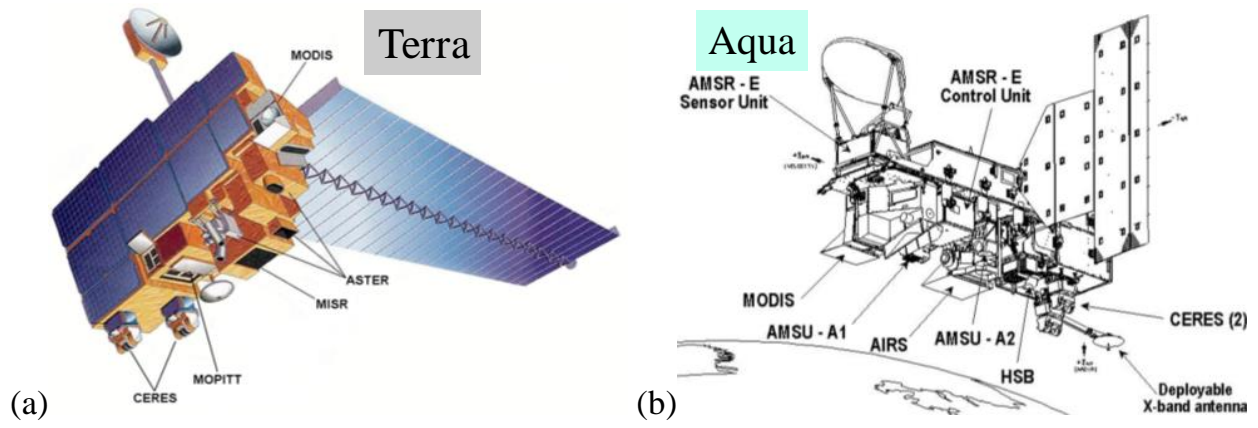

Fig. 2. Schematics of the platforms showing the MODIS sensors mounted on the nadir deck: (a) Terra; (b) Aqua that has AMSR-E sensor mounted on the zenith deck directly above the MODIS sensor.

This paper is arranged as follows. This section briefly introduces the MODIS sensors and the geolocation error detection method. Section 2 summarizes overall geolocation accuracy in MODIS Collection 5 (C5), C6 and C6.1. Sections 3 and 4 details the time series of geolocation uncertainties in C5, C6, and C6.1 for Terra MODIS and Aqua MODIS, respectively. Section 5 adds the new findings in scan-to-scan underlaps and on-orbit measurements of effective focal length. Lastly, Section 6 gives concluding remarks and a plan for the near future. 


\section{MODIS RE-PROCESSING COLLECTIONS AND GEOLOCATION RESULTS SUMMARY}

MODIS calibrated radiances Level-1B data products and geolocation fields data products [16] have improved during the Terra and Aqua missions. When major improvements are ready, re-processing in a new collection begins. In this paper, we present results in three collections, 5, 6 and 6.1. Each collection has the data re-processed from the beginning of the missions, from 24 February 2000 for Terra MODIS and 4 July 2002 for Aqua MODIS. Near the time when re-processing of each collection started, such as in the middle of 2005 for C5, forward-processing in that collection also began, overlapping the previous collection forward-processing. This process is schematically demonstrated in Fig. 3. Fig. 3 also shows the termination time of C5 forward-processing in early 2017, after C6 data products have been available for long enough time (more than 6 years in this case) so that all users have transitioned to using data in the updated collection of data products.

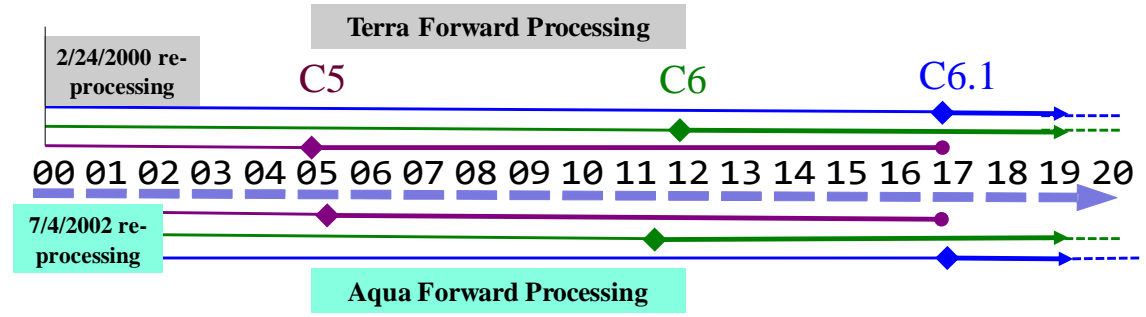

Fig. 3. Illustration of the MODIS re-processing collections. The years are marked in two digits for convenience of viewing in scale from year 2000 to 2020. The upper part is for Terra MODIS re-processing and forward-processing, while the lower part is for Aqua MODIS.

In re-processing, careful analysis has been applied to correct for long-term and within-orbit trends in geolocation biases based on the geolocation errors detected in the previous collection [9]. In forward processing, geolocation biases corrections are predicted about once a year based on a model of the past data. Note that geolocation accuracy values are for MODIS Band 1 through ground control image matching [5,9]. For other bands, users can use the band-to-band registration offsets $[17,18]$ relative to band 1 to determine the geolocation of each band if needed. Note that although the instruments were designed to minimized the band-to-band offsets, in some cases these offsets can be large, e.g. $0.4 \mathrm{~km}$ (at nadir) along-track for Aqua MODIS Band 7 in 2003 [18].

The overall geolocation accuracy assessments for the completed C5 and on-going C6 and C6.1 are listed in Table 1. All three collections have excellent geolocation accuracy with means close to zero and the root-mean-square errors (RMSEs) of $54 \mathrm{~m} \mathrm{(} \mathrm{20 \%} \mathrm{of} \mathrm{a} \mathrm{QKM} \mathrm{band)} \mathrm{or} \mathrm{less.} \mathrm{In} \mathrm{general,} \mathrm{the} \mathrm{biases} \mathrm{in} \mathrm{mean} \mathrm{errors} \mathrm{in} \mathrm{the} \mathrm{track} \mathrm{and} \mathrm{scan} \mathrm{directions} \mathrm{are} \mathrm{gradually}$ reduced from each reprocessing. That is also true for the RMSEs. The improvements seems small for the whole collection or the mission-to-date. However, they result from corrections for larger biases that occurred for short time periods, as we will see in details in Sections $3 \& 4$. Note that the measured geolocation errors are adjusted for scan angle and expressed in nadir equivalent units [5].

Table 1. Each collection's overall MODIS geolocation accuracy in nadir equivalent units.

\begin{tabular}{|c|c|c|c|c|c|c|}
\hline Residuals & Terra C5 & Aqua C5 & Terra C6 & Aqua C6 & Terra C6.1 & Aqua C6.1 \\
\hline Track mean & $-1 \mathrm{~m}$ & $8 \mathrm{~m}$ & $1 \mathrm{~m}$ & $3 \mathrm{~m}$ & $0 \mathrm{~m}$ & $1 \mathrm{~m}$ \\
\hline Scan mean & $5 \mathrm{~m}$ & $2 \mathrm{~m}$ & $0 \mathrm{~m}$ & $2 \mathrm{~m}$ & $0 \mathrm{~m}$ & $0 \mathrm{~m}$ \\
\hline Track RMSE & $44 \mathrm{~m}$ & $48 \mathrm{~m}$ & $43 \mathrm{~m}$ & $46 \mathrm{~m}$ & $43 \mathrm{~m}$ & $46 \mathrm{~m}$ \\
\hline Scan RMSE & $44 \mathrm{~m}$ & $54 \mathrm{~m}$ & $45 \mathrm{~m}$ & $54 \mathrm{~m}$ & $45 \mathrm{~m}$ & $53 \mathrm{~m}$ \\
\hline Data-days & $6097(16.7 \mathrm{y})$ & $5269(14.4 \mathrm{y})$ & $7025(19.2 \mathrm{y})$ & $6214(17.0 \mathrm{y})$ & $7029(19.2 \mathrm{y})$ & $6214(17.0 \mathrm{y})$ \\
\hline Missing days & 134 & 97 & 61 & 10 & 59 & 10 \\
\hline Daily matches & 255 & 220 & 257 & 221 & 257 & 222 \\
\hline
\end{tabular}




\section{TERRA MODIS GEOLOCATION ACCURACY IN C5, C6 \& C6.1}

The Terra MODIS C5 data products were generated until early 2017. Data products in C6 and C6.1 have been re-processed from the beginning of the mission from 24 February 2000 and the forward-processing is on-going (see Section 2).

Fig. 4 shows the time series of Terra MODIS C5 geolocation errors detected expressed in daily means, 16-day global means, 16-day southern hemisphere means, and 16-day northern hemisphere means. The majority of the geolocation uncertainties (means and 16-day standard deviations) are within $+/-50 \mathrm{~m}$, or $20 \%$ of the QKM band resolution in nadir equivalent units. Exceptions are during the periods around 2005 to 2006 in the scan direction, and the periods around 2011 to 2014 in the track direction.

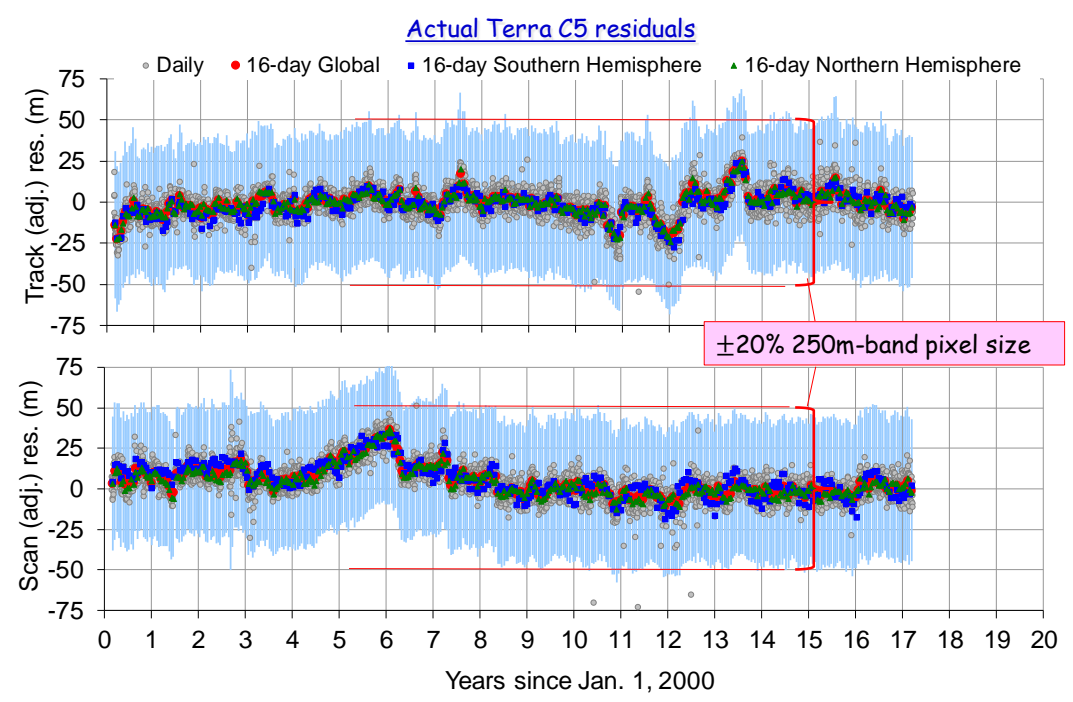

Fig. 4. Time series of Terra MODIS C5 geolocation errors.

Fig. 5 shows the time series of Terra MODIS C6 geolocation errors. The majority of the geolocation uncertainties are within $+/-50 \mathrm{~m}$ in nadir equivalent except the periods around mid-2013 in the track direction.

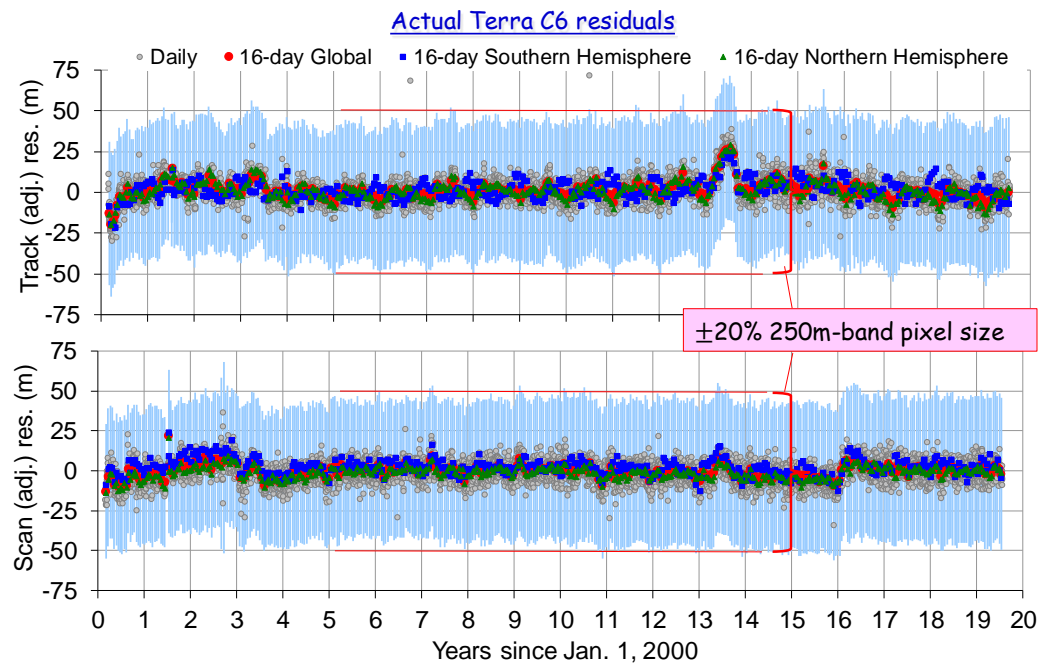

Fig. 5. Time series of Terra MODIS C6 geolocation errors.

Fig. 6 shows the time series of Terra MODIS C6.1 geolocation errors. The majority of the geolocation uncertainties are within $+/-50 \mathrm{~m}$ in nadir equivalent. 


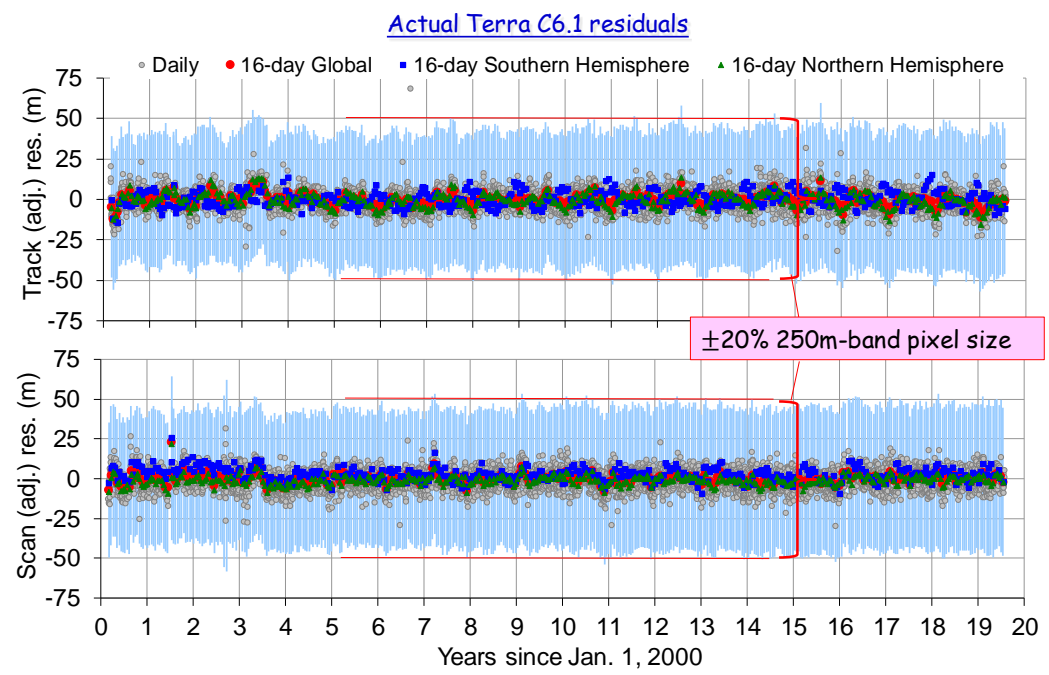

Fig. 6. Time series of Terra MODIS C6.1 geolocation errors.

As we can see from Figures 4, 5 and 6, the geolocation uncertainties are reduced in each progressive re-processing collection. This is accomplished by fine-tuning the instrument-to-spacecraft mounting rotation angles as proxies to the thermal distortion in long-term trends and in annual cycle fluctuations [9], see Fig. 7 as an example for C6.1. Note that at the $705 \mathrm{~km}$ nominal altitude of Terra, a 10 arcsec error in roll or pitch is equivalent to $\sim 34 \mathrm{~m}$ at nadir. The orbital thermal distortion is also modelled using sun angle to the spacecraft travel direction [9]. This correction used C5 data from the beginning of the mission to about 2011 for both C6 and C6.1 (Fig. 8) [9].

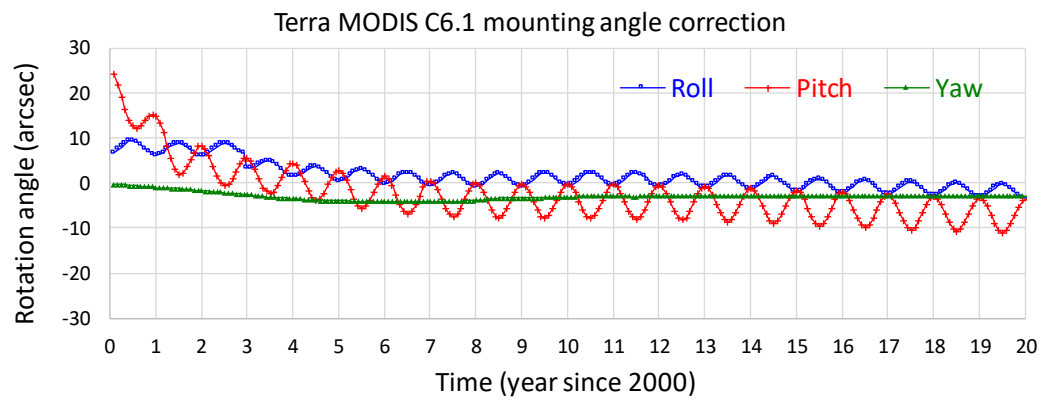

Fig. 7. Time series of instrument-to-spacecraft mounting angle corrections for Terra MODIS C6.1 geolocation.
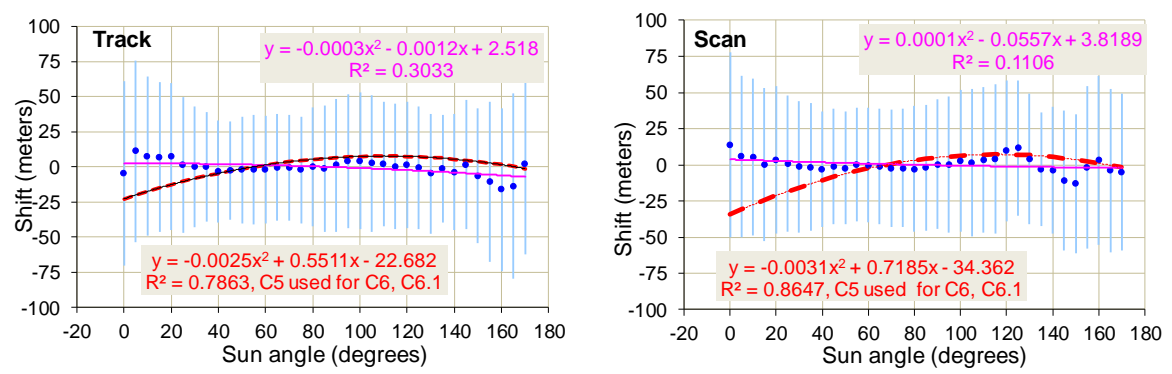

Fig. 8. Correction from $\mathrm{C} 5$ and results in C6.1 of the geolocation errors with respect to sun angle.

To illustrate the effects of corrections shown in Figures 7 and 8, a model [9] is used to "restore" geolocation errors without corrections, as shown in Fig. 9. We can see that, if uncorrected, the geolocation errors would be large at the beginning of the mission in both scan and track direction. In the track direction, the geolocation errors gradually increase. Also, the annual cycle fluctuations are apparent. There are also different geolocation errors in the south and north hemispheres that are corrected by the sun angle functions as shown in Fig. 8 above. 


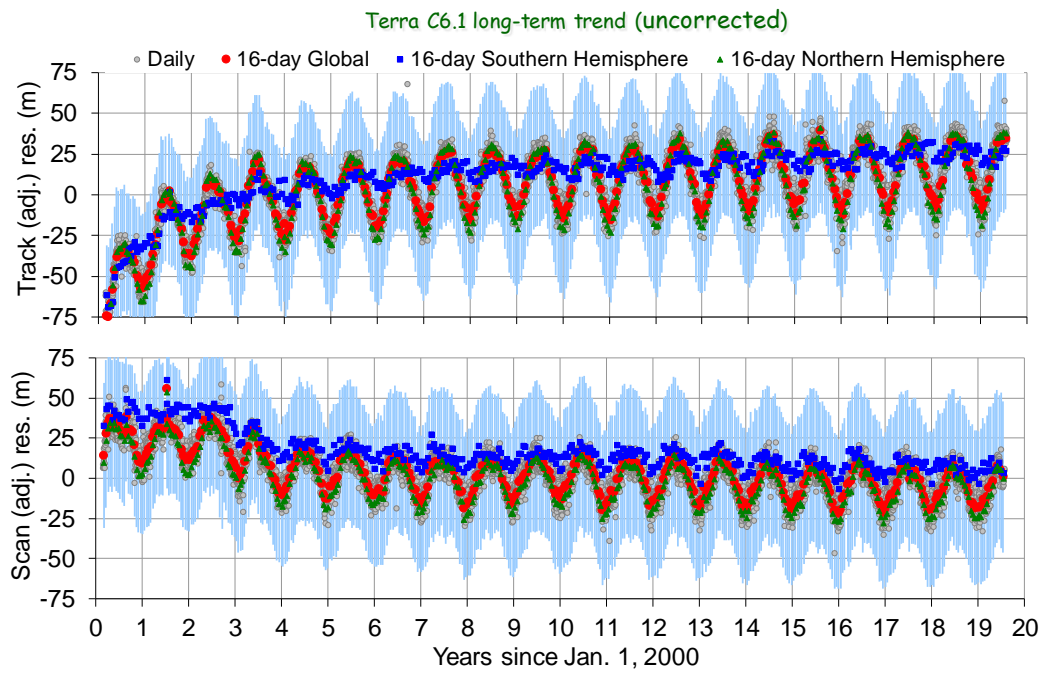

Fig. 9. Illustration of uncorrected geolocation errors for Terra MODIS in C6.1.

\section{AQUA MODIS GEOLOCATION ACCURACY IN C5, C6 \& C6.1}

Similar to Terra MODIS, Aqua MODIS C5 data products were generated until early 2017. Data products in C6 and C6.1 have been re-processed from the beginning of the mission from 4 July 2002 and the forward-processing is on-going (see Section 2).

Fig. 9 shows the time series of Aqua MODIS C5 geolocation errors detected by the CPM expressed in daily means, 16day global means, 16-day southern hemisphere means, and 16-day northern hemisphere means. The majority of the geolocation uncertainties (means and standard deviations) are over $+/-50 \mathrm{~m}$ but within $+/-75 \mathrm{~m}$ nadir equivalent. Further improvements were made in C6 and C6.1.

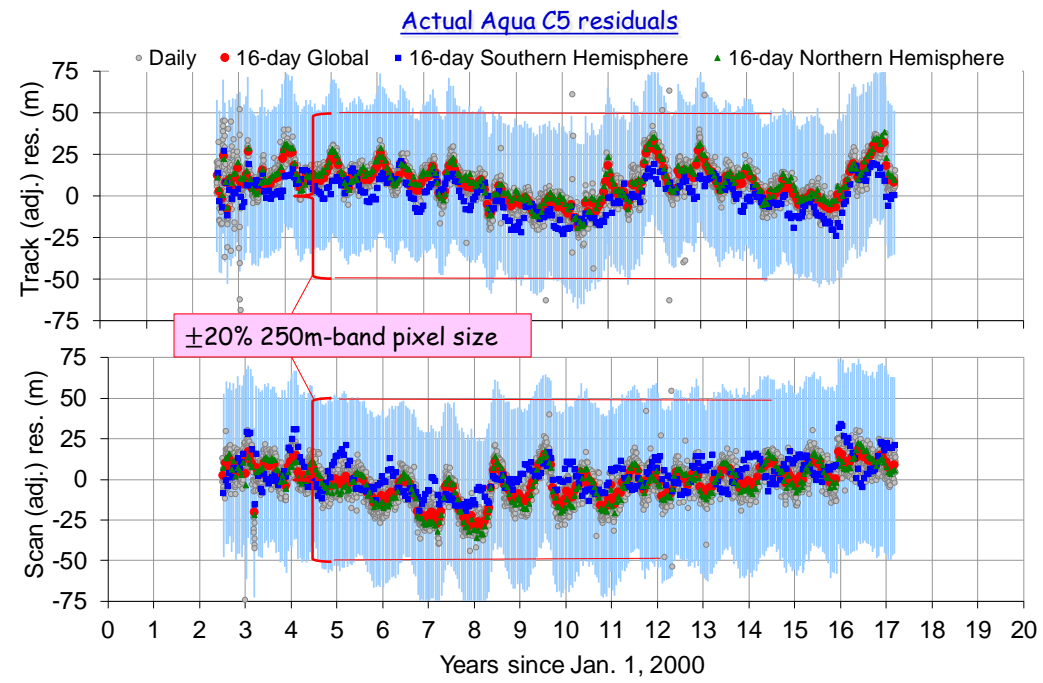

Fig. 9. Time series of Aqua MODIS C5 geolocation errors.

Fig. 10 shows the time series of Aqua MODIS C6 geolocation errors. The geolocation uncertainties before October 2011 have been improved from C5. Unexpected changes were found in the values after October 2011, which were found later to be coincident with the activities of the AMSR-E instrument. 


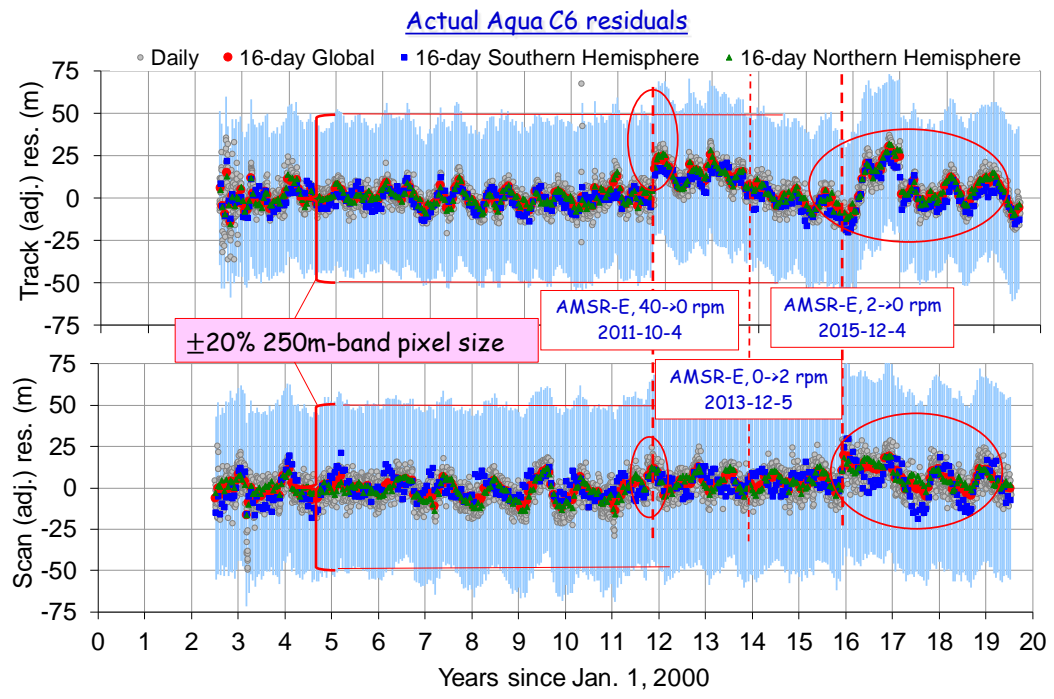

Fig. 10. Time series of Aqua MODIS C6 geolocation errors.

Fig. 11 shows the time series of Aqua MODIS C6.1 geolocation errors. Changes due to the activities of the AMSR-E instrument are corrected. We noticed that the error patterns after 2016, when the antenna of the AMSR-E instrument stopped moving, are different from those before 2016.

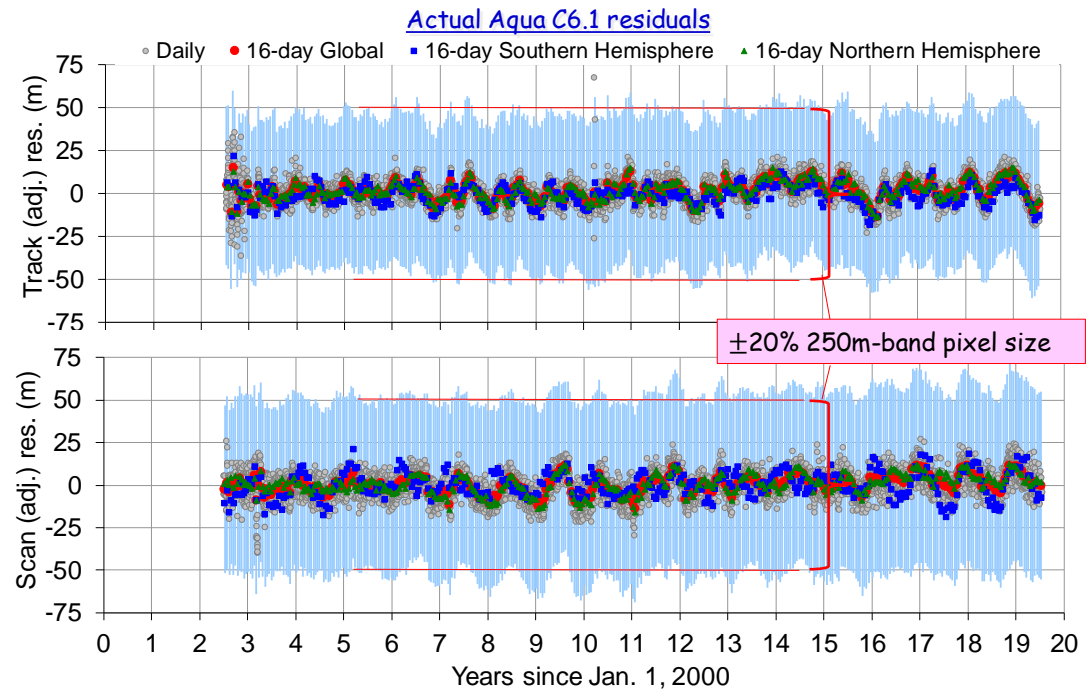

Fig. 11. Time series of Aqua MODIS C6.1 geolocation errors.

As we can see from Figures 9, 10 and 11, the geolocation uncertainties are reduced in each progressive re-processing collection. This is accomplished by fine-tuning the instrument-to-spacecraft mounting rotation angles as proxies to the thermal distortion in long-term trends and in annual cycle fluctuations [9], see Fig. 12 as an example for C6.1. The orbital thermal distortion is also modelled using sun angle to the spacecraft travel direction [9]. This correction used C5 data from the beginning of the mission to about 2011 for both C6 and C6.1 (Fig. 13).

The activities of AMSR-E interrupted the long-term trending pattern. The corrections are adjusted accordingly, as shown in Fig. 12. 


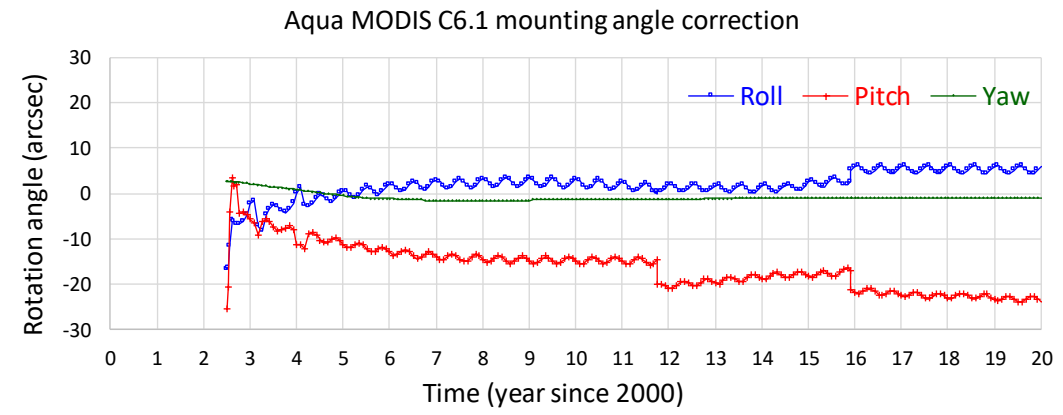

Fig. 12. Time series of instrument-to-spacecraft mounting angle corrections for Aqua MODIS C6.1 geolocation.
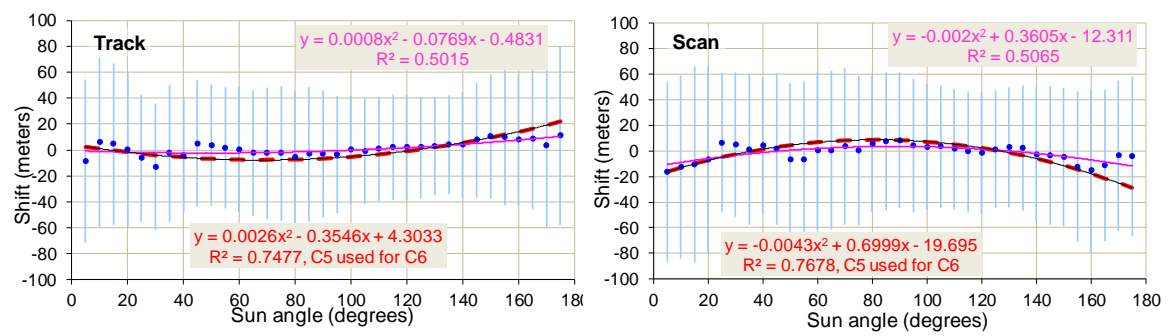

Fig. 13. Correction from C5 and results in C6.1 of the geolocation errors with respect to sun angle.

To illustrate the effects of corrections shown in Figures 12 and 13, a model is used to "restore" geolocation errors without corrections, as shown in Fig. 14. We can see that, if uncorrected, the geolocation errors would be large at the beginning of the missing in both scan and track direction. In the track direction, the geolocation errors gradually increases. The activities of AMSR-E changed the error patterns. Note that in Fig. 11, there remains an annual trend and small hemispherical differences (correlated with sun angle) that may be removed in future reprocessing (C7).

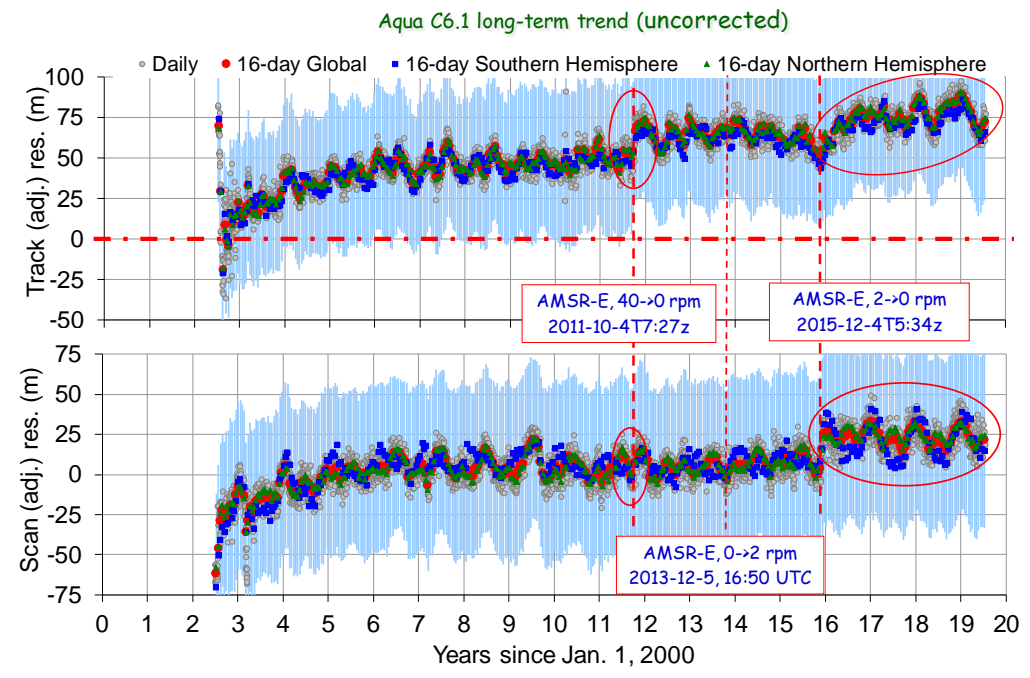

Fig. 14. Illustration of uncorrected geolocation errors for Aqua MODIS in C6.1.

\section{SCAN-TO-SCAN UNDERLAPS AND EFFECTIVE FOCAL LENGTH}

Recently, gaps (underlaps or under-sampling) between VIIRS scans were found during test data analysis of the VIIRS instrument that will be flown on the second Joint Polar Satellite System (JPSS-2) platform with known ephemeris data from Suomi National Polar-orbiting Partnership (S-NPP) [19, 20]. Subsequently it was found that these gaps also exist in MODIS on both the Terra and Aqua platforms. 
The root cause was discovered to be in the calculation of the sub-satellite ground speed in the track direction because the earth rotation speed not taken into account. This ground speed is integral to the design of the instruments like MODIS and VIIRS and has an impact on the focal length, scan period, detector size, focal plane layout and band readout timing.

The maximum size of the gaps is $\sim 100 \mathrm{~m}$ for Terra MODIS at nadir near $15^{\circ} \mathrm{N}$ in both ascending and descending orbits (Fig. 15). The gaps closes at about $60^{\circ} \mathrm{N}$ and $20^{\circ} \mathrm{S}$. At its maximum, the gap closes at $\sim+/-90 \mathrm{~km}$ off nadir in the scan direction because of the bow-tie effect (not shown), see [5]. As the gap at nadir decreases in the ground track direction, the gap extent in the scan direction also decreases.

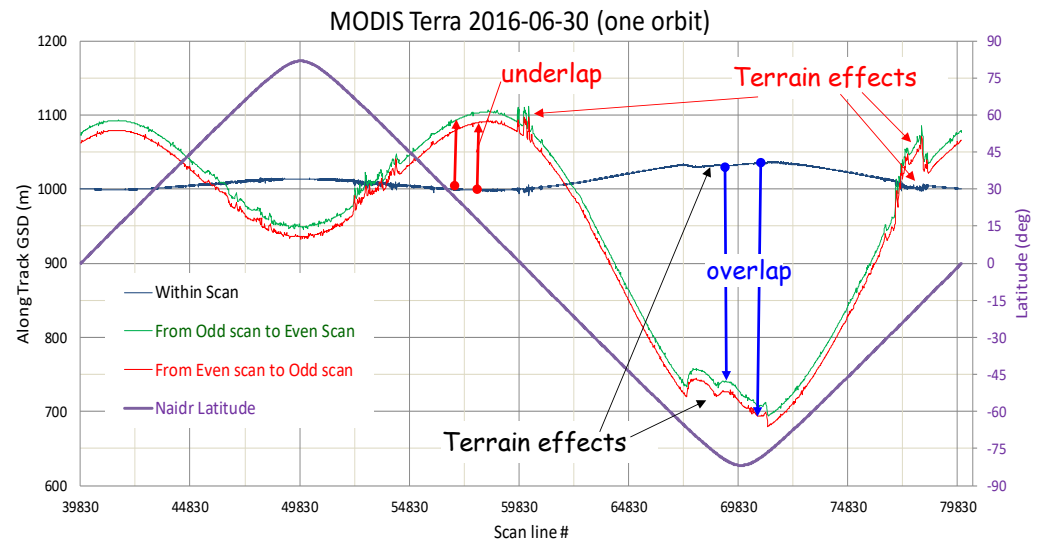

Fig. 15. Scan-to-scan underlaps/overlaps of Terra MODIS at nadir along the ground track of a single orbit. The units for the left yaxis are along-track Ground Sample Distance (GSD) in meters.

For Aqua MODIS, the gaps are $~ 170 \mathrm{~m}$ in one pair of scans and about $30 \mathrm{~m}$ in the next pair of scans due to small wedge angle between the mirror sides (Fig. 16). The locations of the maximum gaps are about the same as Terra MODIS at $15^{\circ}$ $\mathrm{N}$ in both ascending and descending orbits. The larger gaps are throughout the whole northern hemisphere, and extend to about $30^{\circ} \mathrm{S}$. The maximum extent in the scan direction is $\sim+/-120 \mathrm{~km}$ (Fig. 17), which decreases as the sub-satellite point moves away from $15^{\circ} \mathrm{N}$.

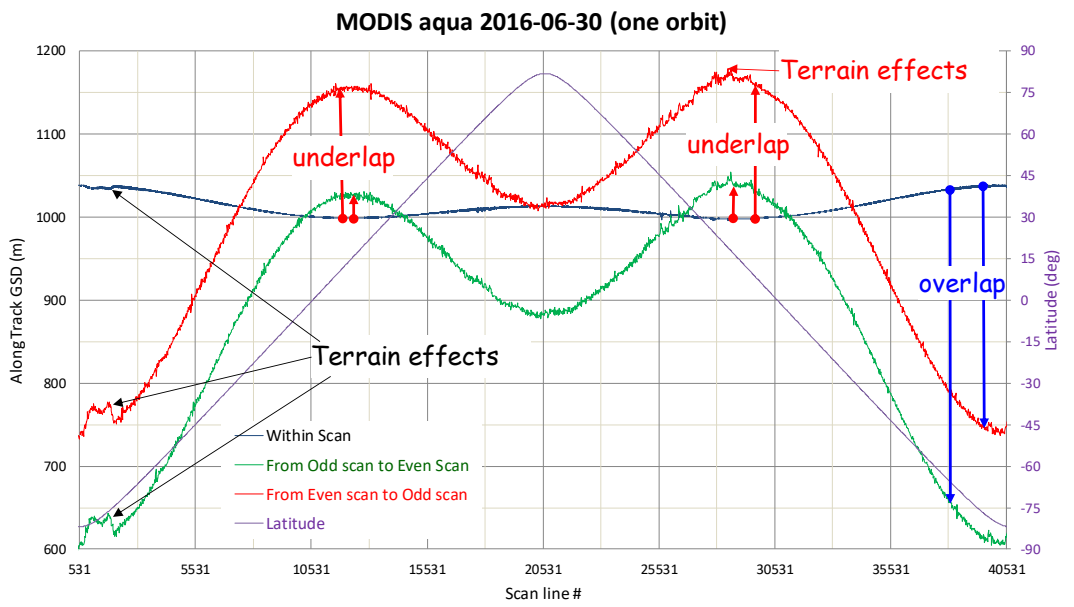

Fig. 16. Scan-to-scan underlaps/overlaps of Aqua MODIS at nadir along the ground track.

Terrain also causes the size of the gaps to vary. Higher terrain enlarges the gaps ( $14 \mathrm{~m}$ per $1 \mathrm{~km}$ terrain height) where they exist or possibility opens up gaps where they do not exist.

Note that there is a large difference in the underlaps in the alternating scans in the Aqua MODIS (Figures 16 and 17). While that difference is small in Terra MODIS (Fig. 15). This is due to differences in scan mirror axis error and mirror axis tilt that were measured based on CPM data in C3 for Terra MODIS and C4 for Aqua MODIS, and were adjusted in C4 for Terra MODIS and C5 for Aqua MODIS and beyond [9]. After those adjustments, the mean geolocation errors in the track direction are close to zero across the scan angles (Fig. 18). Note that the scan angle range runs from $-45^{\circ}$ to $+45^{\circ}$, 
not the full range of $+/-56^{\circ}$. That was decided in the late 1990s when the CPM software was developed, probably in consideration of computing power at the time.

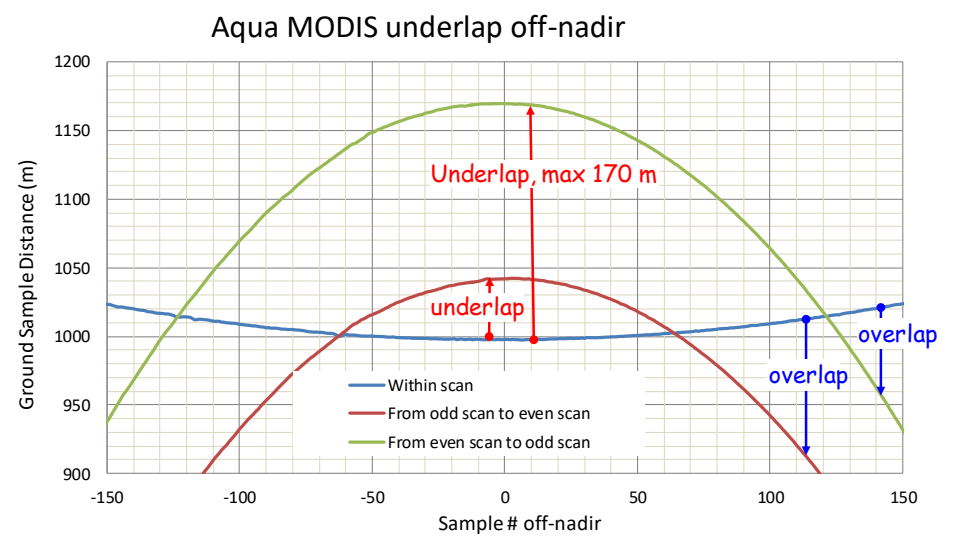

Fig. 17. Scan-to-scan underlaps/overlaps of Aqua MODIS at $15^{\circ} \mathrm{N}$ along the scan direction.

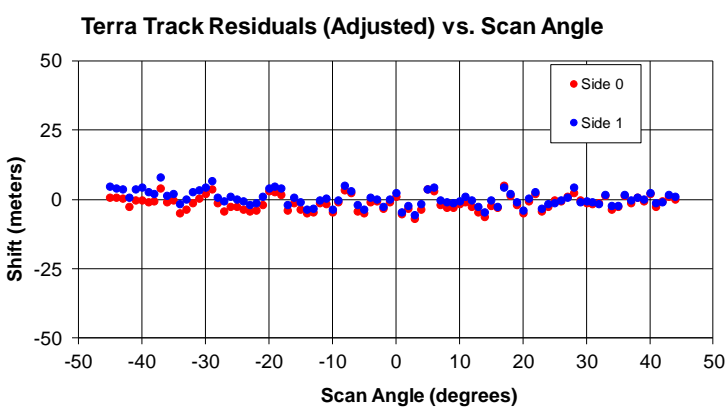

(b)

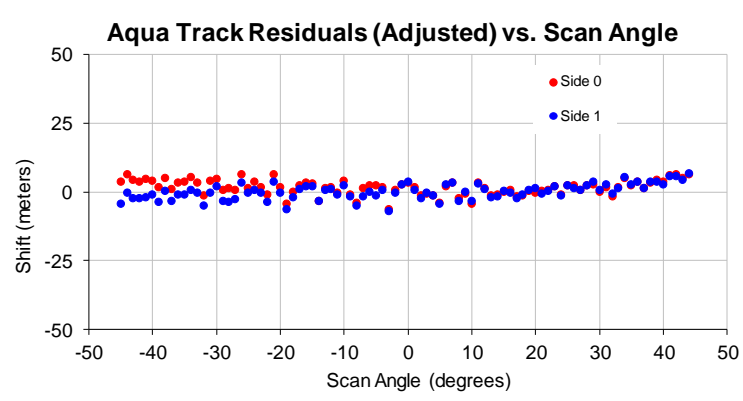

Fig. 18. Mean geolocation errors in the track direction stratified by mirror sides vs. scan angle for (a) Terra, and (b) Aqua MODIS.

The scan-to-scan underlap (the opposite sign of overlap) we show in Figure 15, 16 and 17 may be expressed in the following equation,

$$
\text { Overlap }=n \frac{p}{F} h-\left[V_{E C I}-V_{\text {eartho }} \cos i\right] T
$$

where $\mathrm{F}=$ effective focal length, $\mathrm{p}=$ detector "pitch" interval in the track direction, $\mathrm{n}=\#$ detectors, $\mathrm{h}=$ range from satellite to earth terrain surface, $\mathrm{T}=$ scan mirror rotation period, $\mathrm{i}=$ inclination angle (in ECI) at $98.2^{\circ}, \mathrm{V}_{\mathrm{ECI}}=$ spacecraft ground speed in the inertial frame, $\mathrm{V}_{\text {earth } 0}=$ speed of earth rotation at equator.

When Overlap < 0, underlap occurs. Underlaps occur due to a neglect of earth rotation term in designing the instrument geometric layout.

Since effective focal length (EFL) plays a critical role in overlaps/underlaps, we developed a method [21, 22] to measure the focal length with on-orbit data. Fig. 19 shows the results from a few bands. Those bands on the NIR FPA have shorter focal length on both Terra and Aqua MODIS sensors, indicating smaller underlaps than as shown in Figures 15, 16 and 17. Some bands in Terra MODIS have longer focal length, such as B10 and B12, indicating larger underlaps than that as shown in Fig. 15.

The deviation of EFL not only affects scan-to-scan overlap/underlap. It also affects geolocation, mostly for the pixel at the ends of detector array in the along-track direction. Subsequently, it affects BBR. A $+0.5 \%$ EFL change means $+50 \mathrm{~m}$ change in scan-to-scan underlap or overlap. A $0.5 \%$ difference in EFL in one band can cause the geolocation of the center of the first or last detectors to have a systematic error of $10 \%$ pixel of the finest bands of the $250 \mathrm{~m}$ resolution ( 40 detectors) 
and $2.5 \%$ of the coarsest bands of the $1 \mathrm{~km}$ resolution (10 detectors). Similarly, a $0.5 \%$ difference in EFL in a band pair can cause the BBR of the first or last detectors to have a systematic error of $10 \%$ of the finest bands of the $250 \mathrm{~m}$ resolution ( 40 detectors) and $2.5 \%$ of the coarsest bands of the $1 \mathrm{~km}$ resolution (10 detectors). Because these EFL measurements are recent, they have not been factored into the geometric model currently being used in C6 and C6.1 processing. It is expected that modifying the EFL in the geolocation to match the measured value (of Band 1), will improve the geolocation performance and so will likely be included in a future collection.

Terra MODIS EFL Deviation (\% nominal)

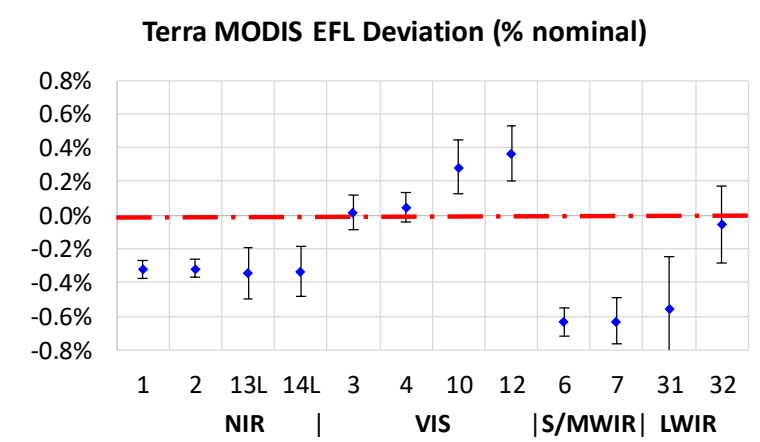

(a)

(b)
Aqua MODIS EFL Deviation (\% nominal)

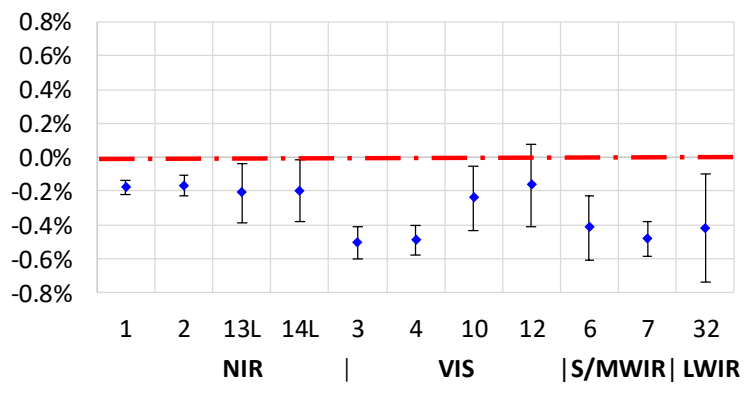

Fig. 19. Summary results of focal length measurements in (a) Terra, and (b) Aqua MODIS.

\section{CONCLUDING REMARKS AND PATHS FORWARD}

The MODIS geolocation products have been re-processed through Collections 5, 6 and 6.1. Forward processing of C6 and C6.1 is on-going with combined data streams in 36+ years (19+ years for Terra MODIS and 17+ years for Aqua MODIS). The geolocation accuracy has improved with each collection, including corrections for some short term geolocation biases and pointing errors induced by stop-go-stop activities of the AMSR-E instrument onboard the Aqua platform. For the latest collection C6.1, the overall measured geolocation accuracy in terms of nadir equivalent root-meansquare errors (RMSEs) is $43 \mathrm{~m}$ in the track direction and $45 \mathrm{~m}$ in the scan direction for Terra MODIS, and $46 \mathrm{~m}$ and $53 \mathrm{~m}$ for Aqua MODIS.

A lesson learned from JPSS-2 VIIRS instrument reaches back to MODIS, in that the scan-to-scan underlaps occur near nadir over the equatorial regions. The largest underlap (gap) occurs at $15^{\circ} \mathrm{N}$ in general and at $90 \mathrm{~m}$ in average, and $170 \mathrm{~m}$ in one pair of scans and 30m in the next pair of scans for Aqua MODIS. The size of these underlaps will increase after the Terra and/or Aqua exits their constellations by lowering the orbit by a few $\mathrm{km}$ but remain operational in the future (around year 2022).

Collection 7 is in the planning stage. The major driver is to change the data format from Version 4 of the Hierarchical Data Format (HDF4) to Version 5 (HDF5). Other changes are expected to be incorporated: (1) updating focal length with recent measurements in the geolocation parameter look-up table (LUT) files; (2) adding geolocation products in $250 \mathrm{~m}$ resolution; (3) refreshing Ground Control Point library; and (4) extending CPM measurements from current $+/-45^{\circ}$ to full scan range of $+/-56^{\circ}$ and search area from current $+/-0.8$ pixels to $+/-2.5$ pixels in the scan and track directions as is done in VIIRS CPM [20].

\section{ACKNOWLEDGEMENTS}

The authors acknowledge the support of the MODIS calibration support team, the MODIS Adaptive Processing System (MODAPS) production team and the MODIS science data support team. This work was performed under the direction of the MODIS Science Team in the Terrestrial Information Systems Laboratory (Code 619) at NASA Goddard Space Flight Center. 


\section{REFERENCES}

[1] https://mcst.gsfc.nasa.gov/mcst-meetings/, accessed 22 August 2019.

[2] https://modis.gsfc.nasa.gov/sci team/meetings/, accessed 22 August 2019.

[3] https://mcst.gsfc.nasa.gov/publications/, accessed 22 August 2019.

[4] https://modis.gsfc.nasa.gov/sci team/pubs/, retrieved 22 August 2019.

[5] R.E Wolfe, M. Nishihama, A.J Fleig, J.A Kuyper, D.P Roy, J.C Storey, F.S Patt. "Achieving sub-pixel geolocation accuracy in support of MODIS land science.” Remote Sensing of Environment, 83 (2002), pp. 31-49.

[6] Glickman, J., Hashmall, J., Natanson, G., Sedlak, J., Tracewell, D. (2003). Earth Observing System (EOS) Aqua launch and attitude support experiences. Flight Mechanics Symposium Proceedings, NASA Goddard Space Flight Center, Greenbelt, Maryland.

[7] Wolfe, R. E. (2006). Chapter 4: MODIS geolocation. Earth Science Satellite Remote Sensing, Geosciences of Springer-Verlag and Tsinghua University Press, 1, pp. 50-71.

[8] R. E. Wolfe, Masahiro Nishihama, (2009), "Trends in MODIS geolocation error analysis." Earth Observing Systems XIV, edited by James J. Butler, Xiaoxiong Xiong, Xingfa Gu Proc. of SPIE Vol. 7452, 74520L, doi: $10.1117 / 12.826598$.

[9] Wolfe, R.E.; Nishihama, M. "Accurate MODIS global geolocation through automated ground control image matching”. In Image Registration for Remote Sensing, Le Moigne, J., Netanyahu, N.S., Eastman, R.D., Eds. Cambridge University Press: Cambridge, 2011, 437-455; doi: 10.1017/CBO9780511777684.022.)..

[10] Salomonson, V. V., Barnes, W. L., Maymon, P. W., Montgomery, H. E., Ostrow, H. (1989). MODIS: Advanced facility instrument for studies of the Earth as a system. IEEE Transactions on Geoscience and Remote Sensing, 27, pp. 145-153.

[11] Nishihama, M., Wolfe, R. E., Solomon, D., Patt, F. S., Blanchette, J., Fleig, A. J., and Masuoka, E. (1997). MODIS Level 1A Earth Location: Algorithm Theoretical Basis Document Version 3.0, SDST-092. Laboratory for Terrestrial Physics, NASA Goddard Space Flight Center, Greenbelt, MD.

[12] https://landsat.gsfc.nasa.gov/about/history/, accessed 22 August 2019.

[13] https://www.usgs.gov/land-resources/national-land-imaging-program, accessed 22 August 2019.

[14] https://www.nasa.gov/mission_pages/terra/spacecraft/index.html, accessed 22 August 2019.

[15] Parkinson, Claire. "2015 Aqua Update". NASA Sounder Science Team Meeting, Greenbelt, Maryland October 1316, 2015, https://airs.jpl.nasa.gov/resources/presentations, accessed 22 August 2019.

[16] https://modis.gsfc.nasa.gov/data/dataprod/, accessed 22 August 2019.

[17] Xiaoxiong, X.; Nianzeng, C.; Barnes, W. Terra MODIS on-orbit spatial characterization and performance. IEEE 832 Transactions on Geoscience and Remote Sensing 2005, 43, 355-365, doi:10.1109/TGRS.2004.840643.

[18] Xiong, X.; Che, N.; Barnes, W.; Xie, Y.; Wang, L.; Qu, J. Status of Aqua MODIS spatial characterization and 834 performance; Proc of SPIE: 2006, 6361, doi: 10.1117/12.687162.

[19] Lin, Guoqing (Gary), Robert E. Wolfe, James C. Tilton (2016), "Trending of SNPP ephemeris and its implications on VIIRS geometric performance", Proc. of SPIE, Vol. 9972, 99721K, doi: 10.1117/12.2239043.

[20] G. Lin and R. E. Wolfe, "JPSS-1 VIIRS at-launch geometric performance," Earth Observing Systems XXI, edited by J. J. Butler, X. Xiong and X. Gu, Proc of SPIE Vol. 9972, 99721L, doi: 10.1117/12.2238804.

[21] Tilton, J. C., R. E. Wolfe, G. Lin, and Z. Yin (2017), "On-orbit measurement of the focal length of the SNPP VIIRS instrument," in Proceedings of the IEEE International Geoscience and Remote Sensing Symposium, Fort Worth, TX, USA, pp. 4056-4059, July 23-28, 2017, doi: 10.1109/IGARSS.2017.8127890.

[22] Tilton, J. C., R. E. Wolfe, G. Lin, and J. Dellomo (2019), "On-Orbit Measurement of the Effective Focal Length and Band-to-Band Registration of Satellite-Borne Whiskbroom Imaging Sensors", IEEE J. Sel. Topics Appl. Earth Observ. And Rem. Sensing, Submitted May 2019. 